\title{
Word Sense Disambiguation using Static and Dynamic Sense Vectors
}

\author{
Jong-Hoon Oh, and Key-Sun Choi \\ Computer Science Division, Dept. of EECS, Korea Advanced Institute of Science \& Technology \\ (KAIST) / Korea Terminology Research Center for Language and Knowledge Engineering \\ (KORTERM), 373-1, Guseong-dong, Yuseong-gu, Daejeon, 305-701, Korea \\ Email: \{rovellia,kschoi\}@world.kaist.ac.kr
}

\begin{abstract}
It is popular in WSD to use contextual information in training sense tagged data. Co-occurring words within a limited window-sized context support one sense among the semantically ambiguous ones of the word. This paper reports on word sense disambiguation of English words using static and dynamic sense vectors. First, context vectors are constructed using contextual words ${ }^{1}$ in the training sense tagged data. Then, the words in the context vector are weighted with local density. Using the whole training sense tagged data, each sense of a target word ${ }^{2}$ is represented as a static sense vector in word space, which is the centroid of the context vectors. Then contextual noise is removed using a automatic selective sampling. A automatic selective sampling method use information retrieval technique, so as to enhance the discriminative power. In each test case, a automatic selective sampling method retrieves $N$ relevant training samples to reduce noise. Using them, we construct another sense vectors for each sense of the target word. They are called dynamic sense vectors because they are changed according to a target word and its context. Finally, a word sense of a target word is determined using static and dynamic sense vectors. The English SENSEVAL test suit is used for this experimentation and our method produces relatively good results.
\end{abstract}

1 'Contextual words' is defined as a list of content words in context.

${ }^{2}$ In this paper, a target word ' $\mathrm{Wt}$ ' is a semantically

\section{Introduction}

It is popular in WSD to use contextual information in training data (Agirre, et al., 199633; Escudero, et al., 2000; Gruber, 1991; Schutze, 1998). Co-occurring words within a limited window-sized context support one sense among the semantically ambiguous ones of the word. The problem is to find the most effective patterns in order to capture the right sense. It is true that they have similar context and co-occurrence information when words are used with the same sense (Rigau, et al., 1997). It is also true that contextual words nearby an ambiguous word give more effective patterns or features than those far from it (Chen, et al., 1998). In this paper, we represent each sense of a word as a vector in word space. First, contextual words in the training sense tagged data $^{4}$ are represented as context vectors. Then,

ambiguous word in a given context of ' $\mathrm{Wt}$ '. This context may consist of several sentences and it is represented by 'contextual words'.

3 Agirre et al., (1996) defines a term 'conceptual density' based on how many nodes are hit between WordNet node and target words+contexts. Unlike 'Conceptual density', 'local density' used in this paper does not use any semantic net like WordNet but use only the contextual words surrounding the given target word..

${ }^{4}$ In this paper, the English SENSEVAL-2 data for the lexical sample task is used as training sense tagged data. It is sampled from BNC-2, the Penn Treebank (comprising components from the Wall Street Journal, Brown, and IBM manuals) and so on. All items in the lexical sample are specific to one word class; noun, verb or adjective. Training sense tagged data is composed of training samples that support a certain sense of a target word. They contain 
the words in the context vector are weighted with local density. Then, each sense of a target word can be represented as a sense vector, which is the centroid of the context vectors in word space.

However, if training samples contain noise, it is difficult to capture effective patterns for WSD (Atsushi, et al., 1998). Word occurrences in the context are too diverse to capture the right pattern for WSD. It means that the dimension of contextual words will be very large when we will use all words in the training samples for WSD. To avoid the problems, we use an automatized hybrid version of selective sampling that will be called "automatic selective sampling". This automatization is based on cosine similarity for the selection. For a given target word and its context, this method retrieves $\mathrm{N}$-best relevant training samples using the cosine similarity. Using them, we can construct another sense vectors for each sense of the target word. The relevant training samples are retrieved by comparing cosine similarities between given contexts and indexed context vectors of training samples. The 'automatic selective sampling' method makes it possible to use traning samples which have higher discriminative power.

This paper is organized as follows: section 2 shows details of our method. Section 3 deals with experiments. Conclusion and future works are drawn in sections 4 .

\section{Word Sense Disambiguation Method}

\subsection{Overall System Description}

Figure 1 shows the overall system description. The system is composed of a training phase and a test phase. In the training phase, words in the limited context window of training samples, which contains a target word and its sense, are extracted and the words are weighted with local density concept (section 2.2). Then, context vectors, which represent each training sample, are indexed and static sense vectors for each

a target word, its sense and its context. But the sense of contexual words is not annotated in the training samples (SENSEVAL-2, 2001) sense are constructed. A static sense vector is the centroid of context vectors of training samples where a target word is used as a certain sense (section 2.3). For example, two sense vectors of 'bank' can be constructed using context vectors of training samples where 'bank' is used as 'business establishment' and those where 'bank' is used as 'artificial embankment'. Each context vector is indexed for 'automatic selective sampling'.

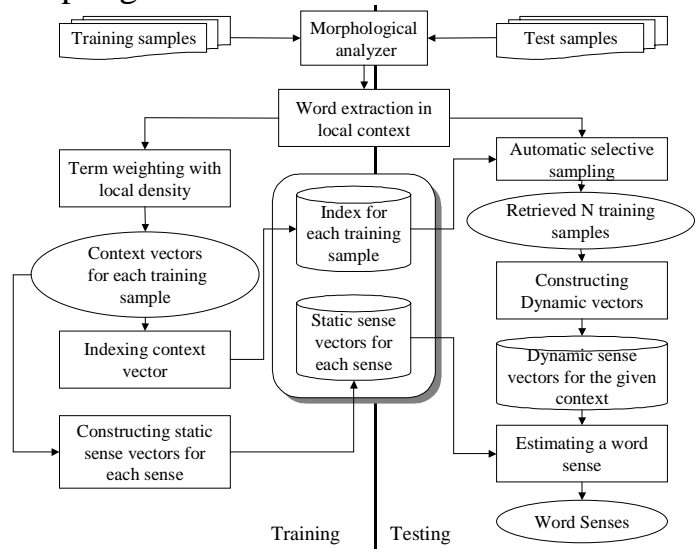

Fig. 1 The overall system description

In the test phase, contextual words are extracted with the same manner as in the training phase (section 2.5). Then, the 'automatic selective sampling' module retrieves top- $N$ training samples. Cosine similarity between indexed context vectors of training samples, and the context vector of a given test sample provides relevant training samples. Then we can make another sense vectors for each sense using the retrieved context vectors. Since, the sense vectors produced by the automatic selective sampling method are changed according to test samples and their context, we call them dynamic sense vectors in this paper (section 2.4) (Note that, the sense vectors produced in the training phase are not changed according to test samples. Thus, we call them static sense vectors.)

The similarities between dynamic sense vectors, and a context vector of a test sample, and those between static sense vectors and the context vector of the test sample are estimated by cosine measure. The sense with the highest similarity is selected as the relevant word sense.

Our proposed method can be summarized as follows

- Training Phase 
1) Constructing context vectors using contextual words in training sense tagged data.

2) Local density to weight terms in context vectors.

3) Creating static sense vectors, which are the centroid of the context vectors.

- Test Phase

1) Constructing context vectors using contextual words in test data.

2) Automatic selective sampling of training vectors in each test case to reduce noise.

3) Creating dynamic sense vectors, which are the centroid of the training vectors for each sense.

4) Estimating word senses using static and dynamic sense vectors

\subsection{Representing Training Samples as a Context Vector with Local Density}

In WSD, context must reflect various contextual characteristics ${ }^{5}$. If the window size of context is too large, the context cannot contain relevant information consistently (Kilgarriff et al., 2000). Words in this context window ${ }^{6}$ can be classified into nouns, verbs, and adjectives. The classified words within the context window are assumed to show the co-occurring behaviour with the target word. They provide a supporting vector for a certain sense. Contextual words nearby a target word give more relevant information to decide its sense than those far from it. Distance from a target word is used for this purpose and it is calculated by the assumption that the target words in the context window have the same sense (Yarowsky, 1995).

Each word in the training samples can be weighted by formula (1). Let $W_{i j}\left(t_{k}\right)$ represent a weighting function for a term $t_{k}$, which appears in the $j^{\text {th }}$ training sample for the $i^{t h}$ sense, $t f_{i j k}$

5 POS, collocations, semantic word associations, subcategorization information, semantic roles, selectional preferences and frequency of senses are useful for WSD (Agirre et al., 2001).

6 Since, the length of context window was considered when SENSEVAL-2 lexical sample data were constructed, we use a training sample itself as context window. represent the frequency of a term $t_{k}$ in the $j^{\text {th }}$ training sample for the $i^{\text {th }}$ sense, $d f_{i k}$ represent the number of training samples for the $i^{\text {th }}$ sense where a term $t_{k}$ appears, $D_{i j k}$ represent the average distance of a term $t_{k}$ from the target word in the $j^{\text {th }}$ training sample for the $i^{\text {th }}$ sense, and $N_{i}$ represent the number of training samples for the $i^{\text {th }}$ sense.

$$
W_{i j}\left(t_{k}\right)=\frac{Z_{i j k}}{Z_{i j}}
$$

where,

$$
\begin{aligned}
& Z_{i j k}=\left(t f_{i j k} \times \frac{1}{\sqrt{D_{i j k}}} \times \frac{d f_{i k}}{D F_{k}} \times \frac{N}{N_{i}}\right) \\
& N=\sum^{\text {all_senses }} N_{i}, \quad D F_{k}=\sum^{\text {all_senses }} d f_{i k} \\
& Z_{i j}=\sqrt{\sum_{k=1}^{\# \text { of_term }}\left(Z_{i j k}\right)^{2}}
\end{aligned}
$$

In formula (1), $\mathrm{Z}$ is a normalization factor, which forces all values of $W_{i j}\left(t_{k}\right)$ to fall into between 0 and 1, inclusive (Salton et al., 1983). Formula (1) is a variation of tf-idf. We regard each training sample as indexed documents, which we want to retrieve and a test sample as a query in information retrieval system. Because we know a target word in training samples and test samples, we can restrict search space into training samples, which contain the target word when we find relevant samples. We also take into account distance from the target word.

$D_{i j k}$ and $d f_{i k}$ in formula (1) support a local density concept. In this paper, 'local density' of a target word ' $W t$ ' is defined by the density among contextual words of ' $W t$ ' in terms of their in-between distance and relative frequency. First, the distance factor is one of the important clues because contextual words surrounding a target word frequently support a certain sense: for example, 'money' in 'money in a bank' .

Second, if contextual words frequently co-occur with a target word of a certain sense, they may be a strong evidence to decide what word sense is correct. Therefore, contextual words, which more frequently appear near a target word and appear with a certain sense of a target word, have a higher local density.

With the local density concept, context of training samples can be represented by a vector 
with context words and their weight, such that $\left(w_{i j}\left(t_{1}\right), w_{i j}\left(t_{2}\right), \ldots, w_{i j}\left(t_{n}\right)\right)$. When $W_{i j}\left(t_{k}\right)$ is 1 , it means that $t_{k}$ is strong evidence for the $i^{\text {th }}$ sense. ( $Z_{i j k}$ are much larger than others.)

\subsection{Constructing Static Sense Vectors}

Now, we can represent each training sample as context vectors using contextual words such that $v_{i j}=\left(w_{i j}\left(t_{1}\right), w_{i j}\left(t_{2}\right), \ldots ., w_{i j}\left(t_{n}\right)\right)$ where $v_{i j}$ represents a context vector of the $j^{\text {th }}$ training sample for the $i^{\text {th }}$ sense and $w_{i j}\left(t_{k}\right)$ is the weight of a term $t_{k}$ calculated by formula (1).

$S V_{i}=\frac{\sum_{j=1}^{\left|N_{i}\right|} v_{i j}}{\left|N_{i}\right|}$

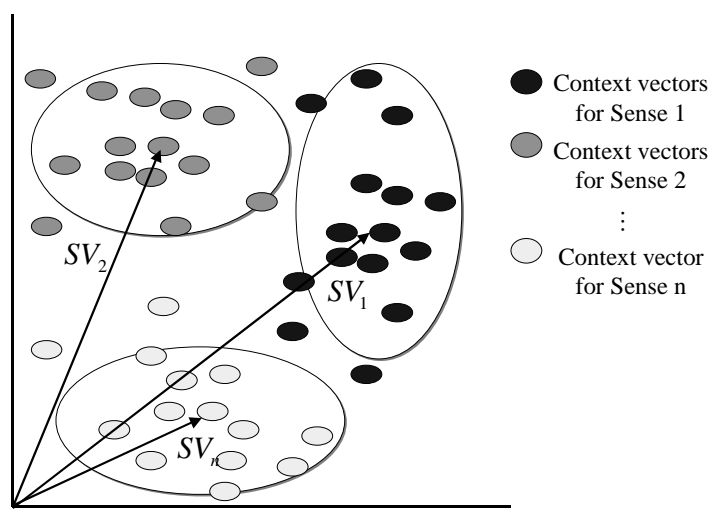

Fig.2 A graphical representation of static sense vectors

Throughout clustering the context vectors, each sense can be represented as sense vectors. Let $N_{i}$ represent the number of training samples for the $i^{t h}$ sense, and $v_{i j}$ represent the context vector of the $j^{\text {th }}$ training sample for the $i^{\text {th }}$ sense. The static sense vector for the $i^{\text {th }}$ sense, $S V_{i}$, can be represented by formula (2) (Park, 1997). In formula (2), $S V_{i}$ is the centroid of context vectors of training samples for the $i^{\text {th }}$ sense as shown in figure 2. In figure 2, there are $n$ senses and context vectors, which represent each training sample. We can categorize each context vector according to a sense of a target word. Then, each sense vectors are acquired using formula (2). Because the sense vectors are not changed according to test samples, we call them a static sense vector in this paper (note that sense vectors, which we will describe in section 2.4, are changed depending on the context of test samples).

\subsection{Automatic selective sampling: Dynamic Sense Vectors}

It is important to capture effective patterns and features from the training sense tagged data in WSD. However, if there is noise in the training sense tagged data, it makes difficult to disambiguate word senses effectively. To reduce its negative effects, we use a automatic selective sampling method using cosine similarity. Figure 3 shows the process of a automatic selective sampling method. The upper side shows retrieval process and the lower side shows a graphical representation of dynamic sense vectors.
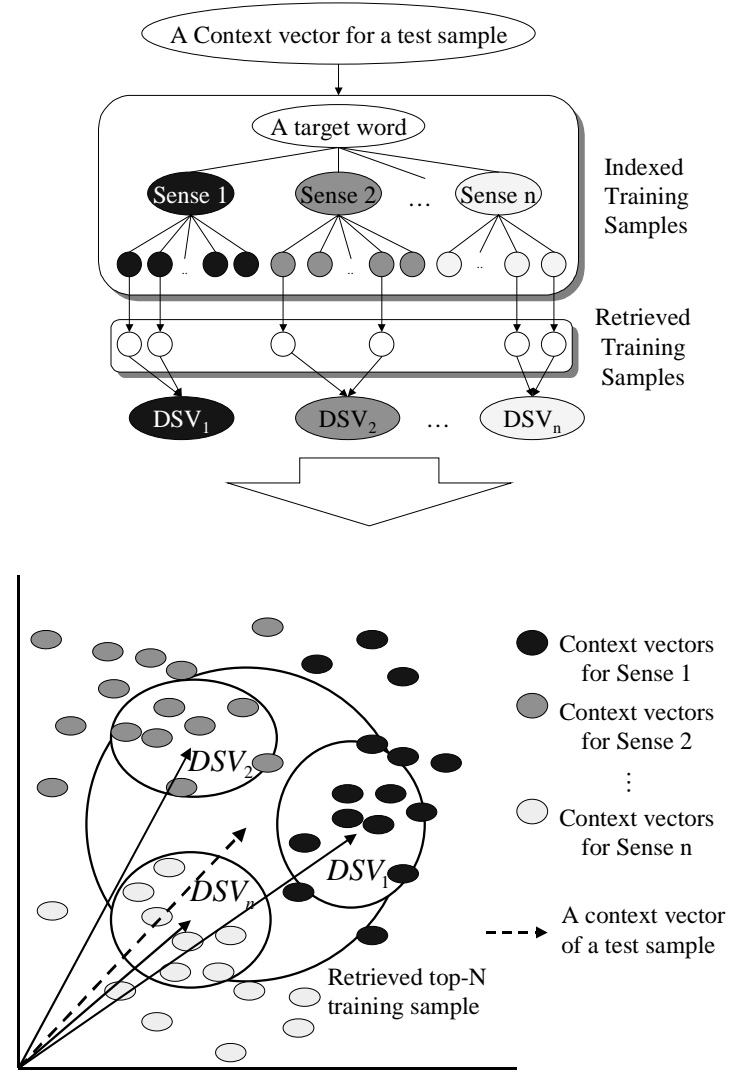

Fig. 3 A graphical representation of an automatic selective sampling method

For example, let 'bank' have two senses ('business establishment', 'artificial embankment'). Now, there are indexed training samples for the two senses. Then top- $N$ training samples can be acquired for a given test sample containing a target word 'bank'. The retrieved 
training samples can be clustered as Dynamic Sense Vectors according to a sense of their target word. Since, the sense vectors produced by a automatic selective sampling method are changed according to the context vector of a test sample, we call them dynamic sense vectors in this paper.

Let $R T_{i}$ represent the number of training samples for the $i^{\text {th }}$ sense in the retrieved top-N, and $v_{i j}$ represent a context vector of the $j^{\text {th }}$ training sample for the $i^{\text {th }}$ sense in the top- $N$. The dynamic sense vector for the $i^{\text {th }}$ sense of a target word, $D S V_{i}$, is formulated by formula (3). In formula (3), $D S V_{i}$ means the centroid of the retrieved context vectors of training samples for the $i^{\text {th }}$ sense as shown in the lower side of figure. 3

$$
D S V_{i}=\frac{\sum_{j=1}^{\left|R T_{i}\right|} v_{i j}}{\left|R T_{i}\right|}
$$

\subsection{Context Vectors of a Test Sample}

Contextual words in a test sample are extracted as the same manner as in the training phase. The classified words in the limited window size nouns, verbs, and adjectives - offer components of context vectors. When a term $t_{k}$ appears in the test sample, the value of $t_{k}$ in a context vector of the test sample will be 1 , in contrary, when $t_{k}$ does not appear in the test sample, the value of $t_{k}$ in a context vector of the test sample will be 0 . Let contextual words of a test sample be 'bank', 'river' and 'water', and dimension of context vector be ('bank', 'commercial', 'money', 'river', 'water'). Then we can acquire a context vector, $\mathrm{CV}=(1,0,0,1,1)$, from the test sample. Henceforth we will denote $C V_{i}$ as a context vector for the $i^{\text {th }}$ test sample.

\subsection{Estimating a Word Sense: Comparing Similarity}

We described the method for constructing static sense vectors, dynamic sense vectors and context vectors of a test sample. Next, we will describe the method for estimating a word sense using them. The similarity in information retrieval area is the measure of how alike two documents are, or how alike a document and a query are. In a vector space model, this is usually interpreted as how close their corresponding vector representations are to each other. A popular method is to compute the cosine of the angle between the vectors (Salton et al., 1983). Since our method is based on a vector space model, the cosine measure (formula (4)) will be used as the similarity measure.

Throughout comparing similarity between $S V_{i}$ and $C V_{j}$ and between $D S V_{i}$ and $C V_{j}$ for the $i^{t h}$ sense and the $j^{\text {th }}$ test sample, we can estimate the relevant word sense for the given context vector of the test sample. Formula (5) shows a combining method of $\operatorname{sim}\left(S V_{i}, C V_{j}\right)$ and $\operatorname{sim}\left(D S V_{i}, C V_{j}\right)$. Let $C V_{j}$ represent the context vector of the $j^{\text {th }}$ test sample, $s_{i}$ represent the $i^{\text {th }}$ sense of a target word, and $\operatorname{Score}\left(s_{i}, C V_{j}\right)$ represent score between the $i^{\text {th }}$ sense and the context vector of the $j^{\text {th }}$ test sample.

$$
\operatorname{sim}(v, w)=\frac{\sum_{i=1}^{N} v_{i} w_{i}}{\sqrt{\sum_{i=1}^{N} v_{i}^{2} \sum_{i=1}^{N} w_{i}^{2}}}
$$

where, $N$ represents the dimension of the vector space, $v$ and $w$ represent vectors.

$$
\begin{aligned}
& \underset{s_{i}}{\arg \max } \operatorname{Score}\left(s_{i}, C V_{j}\right)= \\
& \lambda \times \operatorname{sim}\left(S V_{i}, C V_{j}\right)+ \\
& (1-\lambda) \times \operatorname{sim}\left(D S V_{i}, C V_{j}\right)
\end{aligned}
$$

where $\lambda$ is a weighting parameter.

Because the value of cosine similarity falls into between 0 and 1 , that of $\operatorname{Score}\left(s_{i}, C V_{j}\right)$ also exists between 0 and 1 . When similarity value is 1 it means perfect consensus, in contrary, when similarity value is 0 it means there is no part of agreement at all. After all, the sense having maximum similarity by formula (5) is decided as the answer.

\section{Experiment}

\subsection{Experimental Setup}

In this paper, we compared six systems as follows.

- The system that assigns a word sense which appears most frequently in the training samples (Baseline)

- The system by the Naïve Bayesian method (A) (Gale, et al., 1992) 
- The system that is trained by co-occurrence information directly without changing. (only with term frequency) (B)

- The system with local density and without automatic selective sampling (C)

- The system with automatic selective sampling and without local density (D)

- The system with local density and automatic selective sampling (E)

System A was used to compare our method with the other method. System B, C, D, and E will show the performance of each component in our proposed method. To evaluate performance in the condition of 'without local density (system B and D)', we weight each word with its frequency in the context of training samples.

The test suit used is the English lexical samples released for SENSEVAL-2 in 2001. This test suit supplies training sense tagged data and test data for noun, verb and adjective (SENSEVAL-2, 2001).

Cross-validation on training sense tagged data is used to determine the parameters $-\lambda$ in formula (5) and top- $N$ in constructing dynamic sense vectors. We divide training sense tagged data into ten folds with the equal size, and determine each parameter, which makes the best result in average from ten-fold validation. The values, we used, are $\lambda=0.2$, and $N=50$.

The results were evaluated by precision rates (Salton, et al., 1983). The precision rate is defined as the proportion of the correct answers to the generated results.

\subsection{Experimental Results}

\begin{tabular}{|l|l|l|l|l|}
\hline & Noun & Verb & Adjective & Total \\
\hline \hline Baseline & $50.97 \%$ & $40.34 \%$ & $58.04 \%$ & $47.60 \%$ \\
\hline A & $44.04 \%$ & $32.48 \%$ & $43.43 \%$ & $39.09 \%$ \\
\hline B & $24.33 \%$ & $21.31 \%$ & $26.92 \%$ & $23.50 \%$ \\
\hline C & $44.44 \%$ & $33.81 \%$ & $45.38 \%$ & $40.15 \%$ \\
\hline D & $65.47 \%$ & $49.64 \%$ & $66.84 \%$ & $59.09 \%$ \\
\hline E & $66.89 \%$ & $53.74 \%$ & $70.74 \%$ & $62.07 \%$ \\
\hline
\end{tabular}

Table 1. Experimental results

Table 1 shows experimental results. In the result, all systems and baseline show higher performance on noun and adjective than verb. This indicates that the disambiguation of verb is more difficult than others in this test suit. In analysing errors, we found that we did not consider important information for disambiguating verb senses such as adverbs, which can be used as idioms with the verbs. For example, 'carry out', 'pull out' and so on. It is necessary to handle them for more effective WSD.

System B, C, D, and E show how effective local density and dynamic vectors are in WSD. The performance increase was shown about $70 \%$ with local density (system C) and about $150 \%$ with dynamic vectors (system D), when they are compared with system B - without local density and dynamic vectors. This shows that local density is more effective than term frequency. This also shows that automatic selective sampling of training samples in each test sample is very important.

Combining local density and dynamic vectors (system E), we acquire about $62 \%$ performance. Our method also shows higher performance than baseline and system A (the Naïve Bayesian method) - about 30\% for baseline and about $58 \%$ for system A.

As a result of this experiment, we proved that co-occurrence information throughout the local density and the automatic selective sampling is more suitable and discriminative in WSD. This techniques lead up to $70 \% \sim 150 \%$ performance improvement in the experimentation comparing the system without local density and automatic selective sampling.

\section{Conclusion}

This paper reported about word sense disambiguation for English words using static and dynamic sense vectors. Content words noun, verb, and adjective - in the context were selected as contextual words. Local density was used to weight words in the contextual window. Then we constructed static sense vectors for each sense. A automatic selective sampling method was used to construct dynamic sense vectors, which had more discriminative power, by reducing the negative effects of noise in the training sense tagged data. The answer was decided by comparing similarity. Our method is simple but effective for WSD.

Our method leads up to $70 \sim 150 \%$ precision improvement in the experimentation comparing 
the system without local density and automatic selective sampling. We showed that our method is simple but effective. Our method was somewhat language independent, because our method used only POS information. Syntactic and semantic features such as dependency relations, approximated word senses of contextual words and so on may be useful to improve the performance of our method.

\section{References}

Agirre, E. and G. Rigau (1996) Word Sense Disambiguation using Conceptual Density, Proceedings of 16th International Conference on Computational Linguistics(COLING96), Copenhagen, Denmark.

Agirre, E. and D. Martinez, (2001) Knowledge Sources for Word Sense Disambiguation, Proceedings of the Fourth International Conference (TSD 2001).

Fujii, Atsushi , Kentaro Inui, Takenobu Tokunaga, and Hozumi Tanaka, (1998) Selective Sampling for Example-based Word Sense Disambiguation, Computational Linguistics, 24(4), pp. 573-597.

Escudero, G., L. Màrquez and G. Rigau (2000) Boosting Applied to Word Sense Disambiguation, Proceedings of the 11th European Conference on Machine Learning (ECML 2000) Barcelona, Spain. 2000. Lecture Notes in Artificial Intelligence 1810. R. L. de Mántaras and E. Plaza (Eds.). Springer Verlag.

Gale, William A., Kenneth W. Church, and David Yarowsky (1992) A Method for Disambiguating Word Senses in a Large Corpus. Computers and Humanities, 26, 415-439.

Gruber, T. R. (1991) Subject-Dependent Co-occurrence and Word Sense Disambiguation, Proceedings of 29th Annual Meeting of the Association for Computational Linguistics.

Schutze, Hinrich (1998) Automatic Word Sense Discrimination. Computational Linguistics, 24(1), 97-123.

Chen, Jen Nan and Jason S. Chang (1998) A Concept-based Adaptive Approach to Word Sense Disambiguation, Proceedings of 36th Annual Meeting of the Association for Computational Linguistics and 17th International Conference on Computational Linguistics (COLING/ACL-98) pp 237-243.

Kilgarriff, A. and J. Rosenzweig, (2000) English SENSEVAL: Report and Results, Proceedings of 2nd International Conference on Language Resources \& Evaluation (LREC 2000), Athens.

Park,Y.C (1997) "Building word knowledge for information retrieval using statistical information", Ph.D. thesis, Department of Computer Science, Korea Advanced Institute of Science and Technology.

Rigau, G., J. Atserias and E. Agirre, (1997) Combining Unsupervised Lexical Knowledge Methods for Word Sense Disambiguation, Proceedings of joint 35th Annual Meeting of the Association for Computational Linguistics and 8th Conference of the European Chapter of the Association for Computational Linguistics (ACL/EACL'97), Madrid, Spain.

Salton, G. and M. McGill, (1983) Introduction to Modern Information Retrieval, McGraw-Hill, New York.

SENSEVAL-2 (2001)http://www.sle.sharp.co.uk/ senseval2/

Yarowsky, D. (1995) Unsupervised Word Sense Disambiguation Rivaling Supervised Methods, In Proceedings of the 33rd Annual Meeting of the Association for Computational Linguistics, Cambridge, MA, 189-196. 